\title{
Standing waves for a two-way model system for water waves
}

\author{
Min Chen ${ }^{1}$ and Gérard Iooss ${ }^{2}$ \\ ${ }^{1}$ Department of Mathematics, Purdue University, \\ West Lafayette, IN 47907, USA \\ ${ }^{2}$ Institut Universitaire de France, INLN UMR 6618 CNRS-UNSA \\ 1361 route des Lucioles, F-06560 Valbonne, France
}

April 8, 2004

\begin{abstract}
In this paper, we prove the existence of a large family of non-trivial bifurcating standing waves for a model system which describes two-way propagation of water waves in a channel of finite depth or in the near shore zone. In particular, it is shown that, contrary to the classical standing gravity wave problem on a fluid layer of finite depth, the Lyapunov-Schmidt method applies to find the bifurcation equation. The bifurcation set is formed with the discrete union of Whitney's umbrellas in the three-dimensional space formed with 2 parameters representing the time-period and the wave length, and the average of one of the amplitudes.
\end{abstract}

\section{Introduction}

There are many models for studying weakly nonlinear dispersive water waves in a channel or in the near shore zone. For one-way waves, namely when the wave motion occurs in one-direction, the well known KdV (Korteweg-de Vries) and BBM (Benjamin-Bona-Mahoney) equation are the most studied. For two-way waves, a four parameter class of model equations (which are called Boussinesqtype systems)

$$
\begin{aligned}
\eta_{t}+u_{x}+(u \eta)_{x}+a u_{x x x}-b \eta_{x x t} & =0 \\
u_{t}+\eta_{x}+u u_{x}+c \eta_{x x x}-d u_{x x t} & =0
\end{aligned}
$$

was put forward by Bona, Chen and Saut [3] for small-amplitude and long wavelength gravity waves of an ideal, incompressible liquid. Systems (1) are first-order approximations to the two-dimensional Euler equation in the small parameters $\epsilon_{1}=A / h_{0}$ and $\epsilon_{2}=h_{0}^{2} / L^{2}$, where $h_{0}$ is the depth of water in its quiescent state, $A$ is a typical wave amplitude and $L$ is a typical wavelength. The dependent variables $\eta(x, t)$ and $u(x, t)$, scaled by $h_{0}$ and $c_{0}=\sqrt{g h_{0}}$ respectively 
with $g$ being the acceleration of gravity, represent the dimensionless deviation of the water surface from its undisturbed position and the horizontal velocity at the level of $\theta h_{0}$ of the depth of the undisturbed fluid with $0 \leq \theta \leq 1$, respectively. The coordinate $x$ which measures distance along the channel is scaled by $h_{0}$ and time $t$ is scaled by $\sqrt{h_{0} / g}$. The dispersive parameters $a, b, c$ and $d$ are not independently specifiable parameters, but have to satisfy certain physical relevant conditions [3]. Systems in (1) are not only formally approximations to Euler's equation, but also recently further justified by by Bona, Colin and Lannes (cf. [5]). It was proved that the solution of (2) approximates the solution of Euler's equation with the order of accuracy of the equation (cf. $[7,10,6,1,5])$, namely, for any initial value $\left(\eta_{0}, u_{0}\right) \in H^{\sigma}(\mathbb{R})^{2}$ with $\sigma \geq s \geq 0$ large enough, there exists a unique solution $\left(\eta_{\text {euler }}, u_{\text {euler }}\right)$ of Euler equations, such that

$$
\left\|u-u_{\text {euler }}\right\|_{L^{\infty}\left(0, t ; H^{s}\right)}+\left\|\eta-\eta_{\text {euler }}\right\|_{L^{\infty}\left(0, t ; H^{s}\right)}=O\left(\epsilon_{1}^{2} t, \epsilon_{2}^{2} t, \epsilon_{1} \epsilon_{2} t\right)
$$

for $0 \leq t \leq O\left(\epsilon_{1}^{-1}, \epsilon_{2}^{-1}\right)$.

In this work, attention will be directed to $(x, t)$ - periodic solutions of a system of partial differential equations (which we refer as BBM system since it has certain common properties as the BBM equation)

$$
\begin{aligned}
& \eta_{t}+u_{x}+(\eta u)_{x}-\frac{1}{6} \eta_{x x t}=0, \\
& u_{t}+\eta_{x}+u u_{x}-\frac{1}{6} u_{x x t}=0,
\end{aligned}
$$

which is a member of $(1)$ where $\theta=\sqrt{2 / 3}$. One of the advantages that (2) has over alternative Boussinesq-type systems in (1) (see Bona, Chen \& Saut [3]) is the ease with which it may be integrated numerically. Furthermore, it was proved in [2] and [4] that the initial value problem either for $x \in \mathbb{R}$ or with boundary conditions $(x \in[a, b])$ for $(2)$ is well posed in certain natural function classes.

Since we look for periodic solutions in $(x, t)$, let us introduce the scaled variables $\tilde{x}=\frac{2 \pi \sqrt{6}}{\lambda} x, \tilde{t}=\frac{2 \pi \sqrt{6}}{T} t$, with $T / \sqrt{6}$ and $\lambda / \sqrt{6}$ being the time period and the wave length. One obtains the rescaled BBM system (the tilde is dropped for simplicity in notation)

$$
\begin{aligned}
\eta_{t}+\beta u_{x}-\alpha \eta_{x x t}+\beta(u \eta)_{x} & =0 \\
u_{t}+\beta \eta_{x}-\alpha u_{x x t}+\beta\left(u^{2} / 2\right)_{x} & =0
\end{aligned}
$$

where $\alpha$ and $\beta$ are positive parameters defined by

$$
\alpha=(2 \pi)^{2} / \lambda^{2}, \quad \beta=T / \lambda .
$$

The standing waves, we are looking for are solutions $(\eta, u)$ doubly $2 \pi$ - periodic functions of $(x, t)$, with $u$ odd and $\eta$ even in $x$. This fixes the origin in $x$, but leaves the time shift invariance.

Defining the average of $\eta$ by $A$, we have now a 3 -dimensional parameter space, where only the quarter $\alpha>0, \beta>0$ is physically relevant. We prove 
below (see theorem 4) that, roughly speaking, there is a discrete set of surfaces (Whitney's umbrellas) in the space $(\alpha, \beta, A)$, which constitutes the bifurcation set of standing waves, solutions of the system $(3,4)$. It is worth noting that the situation here is extremely different from the standing gravity waves problem for the classical water waves equations solved for the finite depth case by Plotnikov and Toland [9], and in the infinite depth case by Iooss, Plotnikov and Toland [8]. Indeed, in the present case, we show (see Lemma 3) that there is no small divisor problem and it is possible to adapt a Lyapunov-Schmidt method to reduce the bifurcation problem to a one-dimensional bifurcation equation, after using the $O(2)$ invariance of the system (see below). The precise result is set at Theorem 4.

\section{Study of the linearized operator}

Let us study the linearized system

$$
\begin{aligned}
& \eta_{t}+\beta u_{x}-\alpha \eta_{x x t}=f_{x}, \\
& u_{t}+\beta \eta_{x}-\alpha u_{x x t}=g_{x},
\end{aligned}
$$

with $f$ odd in $x$ and $t$, and $g$ even in $x$ and $t$. Let us write the Fourier series

$$
\begin{aligned}
\eta(x, t) & =\sum_{p \geq 0, q \in \mathbb{Z}} \eta_{p q}(\cos p x) e^{i q t}, \\
u(x, t) & =\sum_{p>0, q \in \mathbb{Z}} u_{p q}(\sin p x) e^{i q t}, \\
f(x, t) & =\sum_{p>0, q \in \mathbb{Z}} f_{p q}(\sin p x) e^{i q t}, \\
g(x, t) & =\sum_{p \geq 0, q \in \mathbb{Z}} g_{p q}(\cos p x) e^{i q t} .
\end{aligned}
$$

Then we get for $p>0, q \in \mathbb{Z}$

$$
\begin{aligned}
& i q\left(1+\alpha p^{2}\right) \eta_{p q}+p \beta u_{p q}=p f_{p q}, \\
& p \beta \eta_{p q}-i q\left(1+\alpha p^{2}\right) u_{p q}=p g_{p q},
\end{aligned}
$$

and for $p=0, q \in \mathbb{Z}$

$$
\begin{aligned}
& \eta_{0 q}=0, \text { when } q \neq 0 \text { and } \\
& \eta_{00} \quad \text { is arbitrary. }
\end{aligned}
$$

Let us define

$$
\Delta(p, q)=q^{2}\left(1+\alpha p^{2}\right)^{2}-p^{2} \beta^{2}
$$

then if $\Delta \neq 0$, we obtain

$$
\begin{aligned}
& \eta_{p q}=-\Delta^{-1} p\left[i q\left(1+\alpha p^{2}\right) f_{p q}+p \beta g_{p q}\right] \\
& u_{p q}=-\Delta^{-1} p\left[p \beta f_{p q}-i q\left(1+\alpha p^{2}\right) g_{p q}\right]
\end{aligned}
$$


and the problem is to give estimates for $\left(\eta_{p q}, u_{p q}\right)$ in terms of $\left(f_{p q}, g_{p q}\right)$ in the case when there exists a pair $\left(p_{0}, q_{0}\right)$ satisfying

$$
q_{0}^{2}\left(1+\alpha p_{0}^{2}\right)^{2}-p_{0}^{2} \beta^{2}=0 .
$$

We first observe that

$$
\Delta(p, q)=\left\{q\left(1+\alpha p^{2}\right)-p \beta\right\}\left\{q\left(1+\alpha p^{2}\right)+p \beta\right\},
$$

hence for $\Delta \neq 0$ we have

$$
\left|\eta_{p q}\right|+\left|u_{p q}\right| \leq \frac{p}{\| q\left|\left(1+\alpha p^{2}\right)-p \beta\right|}\left\{\left|f_{p q}\right|+\left|g_{p q}\right|\right\} .
$$

We have now the following useful precision on the couples $(\alpha, \beta)$ solving (7):

Lemma 1 Given $\alpha$ and $\beta$ positive real numbers, the subset

$$
\Sigma_{(\alpha, \beta)}:=\left\{(p, q) \in \mathbb{N}^{2}, q\left(1+\alpha p^{2}\right)-p \beta=0\right\}
$$

of $\mathbb{N}^{2}$ is either empty, or at most finite. When there exists $\left(p_{0}, q_{0}\right) \in \Sigma_{(\alpha, \beta)}$, then $\left(p_{0}, q_{0}\right)$ is the only element of $\Sigma_{(\alpha, \beta)}$ if one of the following conditions is realized:

i) $\alpha$ is irrational

ii) $\alpha$ is rational and $1 /\left(\alpha p_{0}\right)$ is not an integer, and the numbers $\beta^{2}-4 \alpha q_{j}^{2}$ are not squares of rational numbers for $q_{j}=1,2, \ldots q_{m}, q_{j} \neq q_{0}, q_{m}=[\beta /(2 \sqrt{\alpha})]$.

Proof: i) If $\alpha$ is irrational, then $\beta$ is irrational, rationally related to $\alpha$ by

$$
\beta-\alpha p_{0} q_{0}=q_{0} / p_{0}
$$

Another solution $(p, q) \in \mathbb{N}^{2}$ of the above equation would imply

$$
\alpha\left(p_{0} q_{0}-p q\right)+q_{0} / p_{0}-q / p=0
$$

which implies that $\alpha$ is rational. Hence there is a unique element in $\Sigma_{(\alpha, \beta)}$ when $\alpha$ is irrational.

ii) If $\alpha$ is rational, then $\beta$ is also rational. Set

$$
X=p \sqrt{\alpha}, \quad Y=q \frac{\sqrt{\alpha}}{\beta}
$$

then a solution $(p, q) \in \mathbb{N}^{2}$ of $q\left(1+\alpha p^{2}\right)-p \beta=0$, leads to

$$
Y\left(1+X^{2}\right)-X=0 \text {. }
$$

This leads immediately to $Y \leq \frac{1}{2}$ which yields

$$
q \leq q_{m}=[\beta /(2 \sqrt{\alpha})]
$$


where [.] means the integer part. Hence, the only possible values for $q$ are

$$
q=1,2, \ldots q_{m}
$$

and $q_{0}$ is in this set. For each value $q_{j}$ of $q$ we have

$$
p_{j}^{ \pm}=\frac{\beta}{2 q_{j} \alpha}\left(1 \pm \sqrt{1-4 Y_{j}^{2}}\right)
$$

where

$$
Y_{j}=q_{j} \frac{\sqrt{\alpha}}{\beta} .
$$

A necessary condition for $p_{j}^{ \pm}$to be an integer is that $1-4 Y_{j}^{2}$ is the square of a rational number. This is in particular true for $q_{j}=q_{0}$ since

$$
\begin{aligned}
1-4 Y_{j}^{2} & =\frac{\left(\beta^{2}-4 \alpha q_{j}^{2}\right)}{\beta^{2}} \\
1-4 Y_{0}^{2} & =\frac{\left(\beta^{2}-4 \alpha q_{0}^{2}\right)}{\beta^{2}}=\frac{q_{0}^{2}\left(1-\alpha p_{0}^{2}\right)^{2}}{p_{0}^{2} \beta^{2}},
\end{aligned}
$$

which gives $p_{0}^{+}=1 /\left(\alpha p_{0}\right), \quad p_{0}^{-}=p_{0}$. For $q_{j} \neq q_{0}$ the number $1-4 Y_{j}^{2}$ is in general not the square of a rational, hence $p_{j}^{ \pm}$is not integer.

We then have the following (denote by $\mathbb{N}_{0}$ the set $\mathbb{N} \cup\{0\}$ )

Proposition 2 For all positive parameter values $(\alpha, \beta)$ such that there exists a pair $\left(p_{0}, q_{0}\right) \in \mathbb{N}^{2}$ satisfying $q_{0}\left(1+\alpha p_{0}^{2}\right)-p_{0} \beta=0$, there is at most a finite subset $\Sigma_{(\alpha, \beta)}=\left\{\left(p_{j}, q_{j}\right) ; j=0,1, \ldots N\right\} \subset \mathbb{N}^{2}$, satisfying

$$
q_{j}\left(1+\alpha p_{j}^{2}\right)-p_{j} \beta=0, \quad j=0,1, \ldots N,
$$

and a constant $M>0$ (depending on $(\alpha, \beta)$ ) such that for any pair $(p, q) \in$ $\mathbb{N}_{0} \times \mathbb{Z},(p,|q|) \notin \Sigma_{(\alpha, \beta)}$ and $(p, q) \neq(0,0)$ we have

$$
\frac{p+p^{2}|q|}{|| q\left|\left(1+\alpha p^{2}\right)-p \beta\right|} \leq M
$$

Proof: Let first consider pairs $(p, q)$ satisfying $p|q| \geq 2 \beta / \alpha$, then

$$
|q|\left(1+\alpha p^{2}\right)-p \beta \geq\left\{\begin{array}{c}
p \beta+|q| \\
|q|+\alpha p^{2}|q| / 2
\end{array}\right.
$$

hence

$$
\frac{p+p^{2}|q|}{\| q\left|\left(1+\alpha p^{2}\right)-p \beta\right|} \leq 1 / \beta+2 / \alpha
$$

Now for $p=0, q \neq 0$ we have

$$
\frac{0}{\| q\left|\left(1+\alpha 0^{2}\right)-0 \beta\right|}=0
$$


and for $q=0, p \neq 0$

$$
\frac{p+0}{\left|0\left(1+\alpha p^{2}\right)-p \beta\right|}=1 / \beta
$$

Now the set of pairs $(p, q)$ such that $1 \leq p|q|<2 \beta / \alpha$, is finite, hence a finite bound exists once the denominator does not cancel. Such a situation would imply

$$
|q|\left(1+\alpha p^{2}\right)-p \beta=0
$$

i.e $(p,|q|) \in \Sigma_{(\alpha, \beta)}$.

Remark: Let us give a geometric interpretation of Lemma 1 . In the $(\alpha, \beta)$ plane the equation

$$
q^{2}\left(1+\alpha p^{2}\right)^{2}-p^{2} \beta^{2}=0
$$

defines for a fixed $(p, q) \in \mathbb{N}^{2}$ a couple of straight lines, intersecting at $(\alpha, \beta)=$ $\left(-1 / p^{2}, 0\right)$. Only the line

$$
q\left(1+\alpha p^{2}\right)-p \beta=0
$$

is relevant in the quarter of plane $(\alpha, \beta) \in\left(\mathbb{R}^{+}\right)^{2}$. Lemma 1 shows that if $(\alpha, \beta)$ belongs to such a line for $(p, q)=\left(p_{0}, q_{0}\right)$, then it belongs to at most a finite number of such lines for $(p, q) \in \mathbb{N}^{2}$, this number being one in general. Moreover, in the region $\beta^{2}-4 \alpha<0$ of $\left(\mathbb{R}^{+}\right)^{2}$, there is none of these lines, and in the rest of the quarter plane, the union of this discrete set of lines is not dense.

Let us now introduce the Sobolev spaces

$$
H_{\text {如 }}^{k}=H^{k}(\mathbb{R} / 2 \pi \mathbb{Z})^{2}, \quad H_{\text {响 }}^{k, e}=\left\{w \in H_{\text {吅 }}^{k}, w \text { is even in } x\right\}
$$

and similarly $H_{\text {如 }}^{k, o}=\left\{w \in H_{\text {如 }}^{k}, w\right.$ is odd in $\left.x\right\}$. We also define the operator $\pi_{0}$ by

$$
\left(\pi_{0} g\right)(t)=\frac{1}{2 \pi} \int_{-\pi}^{\pi} g(x, t) d x
$$

and $D_{x}^{-1}$ by

$$
\begin{aligned}
D_{x}^{-1} \cos p x & =p^{-1} \sin p x, p \neq 0 \\
D_{x}^{-1} \sin p x & =-p^{-1} \cos p x, \quad D_{x}^{-1} 1=0 .
\end{aligned}
$$

Notice that the operator $D_{x}^{-1}$ consists in first suppressing the average and then take the primitive which has a 0 average. This guarantees the periodicity of $D_{x}^{-1} f$ for any periodic $f \in L^{2}$. In particular one has for any $f \in H_{\natural}^{1}$

$$
D_{x}^{-1} \partial_{x} f=\partial_{x} D_{x}^{-1} f=\left(1-\pi_{0}\right) f .
$$

We can now show the following

Lemma 3 Assume that $\left(\alpha_{0}, \beta_{0}\right)$ is such that $\Sigma_{\left(\alpha_{0}, \beta_{0}\right)}$ has a unique element $\left(p_{0}, q_{0}\right)$ (see the above lemma 1$)$, then the linear system

$$
\mathcal{L}(\eta, u)=(f, g)
$$


where

$$
\mathcal{L}(\eta, u)=D_{x}^{-1}\left(\eta_{t}+\beta_{0} u_{x}-\alpha_{0} \eta_{x x t}, u_{t}+\beta_{0} \eta_{x}-\alpha_{0} u_{x x t}\right),
$$

has a solution if and only if the compatibility condition

$$
\begin{aligned}
i f_{p_{0} q_{0}}+g_{p_{0} q_{0}} & =0 \\
-i f_{p_{0},-q_{0}}+g_{p_{0},-q_{0}} & =0 \\
\pi_{0} g & =0
\end{aligned}
$$

holds. In such a case for $(f, g) \in H_{\natural \natural}^{k, o} \times H_{\natural \natural}^{k, e}$ (means that $f$ is odd in $x$, and $g$ is even in $x$, both doubly periodic $)$, or if $(f, g)=\left(\phi_{x t}, \psi_{x t}\right)$ with $(\phi, \psi) \in$

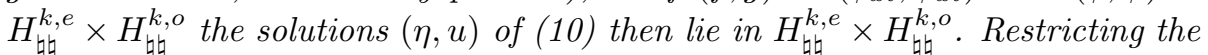
solution $(\eta, u)$ to the subspace $H_{\natural \natural, 0}^{k, e} \times H_{\natural \natural}^{k, o}$ of $H_{\natural \natural}^{k, e} \times H_{\natural \natural}^{k, o}$ such that $\pi_{0}\left(\eta_{t}\right)=0$, the kernel of the linear operator $\mathcal{L}$ is the 3-dimensional subspace spanned by $\xi_{0}=\{1,0\}$ and $\zeta_{0}$ and $\overline{\zeta_{0}}$ where $\mathcal{S} \zeta_{0}=\overline{\zeta_{0}}$ and

$$
\zeta_{0}=\left(e^{i q_{0} t} \cos p_{0} x,-i e^{i q_{0} t} \sin p_{0} x\right) .
$$

Furthermore, the equation (10) has a unique solution $(\eta, u)$, denoted by $\widetilde{\mathcal{L}}^{-1}(f, g)$, which belongs to $H_{\natural \mathrm{\natural}, 0}^{k, e} \times H_{\natural \natural}^{k, o}$ orthogonal in $\left(L_{\mathrm{\natural \natural}}^{2}\right)^{2}$ to $\xi_{0}, \zeta_{0}$ and $\overline{\zeta_{0}}$, and satisfies

$$
\|(\eta, u)\|_{H^{k}} \leq M\|(f, g)\|_{H^{k}} .
$$

Moreover, the equation (10) with $(f, g)=\left(\phi_{x t}, \psi_{x t}\right)$ where $(\phi, \psi) \in H_{\natural \natural}^{k, e} \times H_{\natural \natural}^{k, o}$ leads to a unique solution $(\eta, u)=\widetilde{\mathcal{L}}^{-1}\left(\phi_{x t}, \psi_{x t}\right) \in H_{\natural \natural, 0}^{k, e} \times H_{\natural \natural}^{k, o}$, orthogonal in $\left(L_{\text {如 }}^{2}\right)^{2}$ to $\xi_{0}, \zeta_{0}$ and $\overline{\zeta_{0}}$, which satisfies

$$
\|(\eta, u)\|_{H^{k}} \leq M\|(\phi, \psi)\|_{H^{k}} .
$$

Proof: Notice that equations (3) and (5) imply that $\pi_{0}\left(\eta_{t}\right)=0$. This justifies our restriction to the solutions such that such a condition is realized. We then notice that the system $(5)$ for $(\alpha, \beta)=\left(\alpha_{0}, \beta_{0}\right)$ and with the condition $\pi_{0}\left(\eta_{t}\right)=0$, is equivalent to

$$
\mathcal{L}(\eta, u)=(f, \tilde{g})
$$

where $\tilde{g}=g-\pi_{0} g$ satisfies $\pi_{0} \tilde{g}=0$. Then we have $\tilde{g}_{p q}=g_{p q}$ for $p \neq 0$, hence for $(p,|q|) \neq\left(p_{0}, q_{0}\right)$ and $p>0$

$$
\begin{aligned}
& \eta_{p q}=-\frac{1}{\Delta_{0}(p, q)} p\left[i q\left(1+\alpha_{0} p^{2}\right) f_{p q}+p \beta_{0} \tilde{g}_{p q}\right] \\
& u_{p q}=-\frac{1}{\Delta_{0}(p, q)} p\left[p \beta_{0} f_{p q}-i q\left(1+\alpha_{0} p^{2}\right) \tilde{g}_{p q}\right]
\end{aligned}
$$

where

$$
\Delta_{0}(p, q)=q^{2}\left(1+\alpha_{0} p^{2}\right)^{2}-p^{2} \beta_{0}^{2}
$$

and

$$
\begin{aligned}
\eta_{o q}= & 0, \text { for } q \neq 0, \text { (by construction) } \\
& \eta_{00} \text { arbitrary }
\end{aligned}
$$


and if and only if (11) is satisfied

$$
\begin{aligned}
\eta_{p_{0} q_{0}}=-\frac{i f_{p_{0} q_{0}}}{2 \beta_{0}}+i a, & \eta_{p_{0},-q_{0}}=\frac{i f_{p_{0},-q_{0}}}{2 \beta_{0}}-i b \\
u_{p_{0} q_{0}}=\frac{f_{p_{0} q_{0}}}{2 \beta_{0}}+a, & u_{p_{0},-q_{0}}=\frac{f_{p_{0},-q_{0}}}{2 \beta_{0}}+b
\end{aligned}
$$

where $a$ and $b$ are arbitrary. Orthogonality in $\left(L_{\text {吼 }}^{2}\right)^{2}$ to $\xi_{0}, \zeta_{0}$ and $\overline{\zeta_{0}}$ leads to

$$
\begin{array}{rr}
\eta_{00}=0, & \\
\eta_{p_{0} q_{0}}=-\frac{i f_{p_{0} q_{0}}}{2 \beta_{0}}, & \eta_{p_{0},-q_{0}}=\frac{i f_{p_{0},-q_{0}}}{2 \beta_{0}} \\
u_{p_{0} q_{0}}=\frac{f_{p_{0} q_{0}}}{2 \beta_{0}}, & u_{p_{0},-q_{0}}=\frac{f_{p_{0},-q_{0}}}{2 \beta_{0}} .
\end{array}
$$

The estimate obtained in (8)-(9) leads to $(\eta, u) \in H_{\natural \natural, 0}^{k, e} \times H_{\natural \natural}^{k, o}$ satisfying (12) or (13) as soon as $(f, g) \in H_{\natural \natural}^{k, o} \times H_{\natural \natural}^{k, e}$ or $(\phi, \psi) \in H_{\natural \natural}^{k, e} \times H_{\natural \natural}^{k, o}$, and the compatibility condition (11) is satisfied. This gives the precise range of $\mathcal{L}$. The result on the kernel is a direct consequence of the above formulas.

\section{Bifurcation problem}

Let us introduce the two symmetry linear operators $\mathcal{T}_{\tau}$ and $\mathcal{S}$, for any real $\tau$

$$
\begin{aligned}
\left\{\mathcal{T}_{\tau}(\eta, u)\right\}(x, t) & =(\eta(x, t+\tau), u(x, t+\tau)) \\
\{\mathcal{S}(\eta, u)\}(x, t) & =(\eta(x,-t),-u(x,-t)) .
\end{aligned}
$$

These operators commute with the system $(3,4)$ and we have $\mathcal{T}_{\tau} \mathcal{S}=\mathcal{S} \mathcal{T}_{-\tau}$. It results that the nonlinear system $(3,4)$ possesses a $O(2)$ symmetry associated with the above operators. Let us consider $(3,4)$ for parameter values $(\alpha, \beta)=$ $\left(\alpha_{0}+\nu, \beta_{0}+\mu\right)$, where $\left(\alpha_{0}, \beta_{0}\right)$ is as in the above lemma

$$
\begin{aligned}
\eta_{t}+\beta_{0} u_{x}-\alpha_{0} \eta_{x x t}+\left(\left(\beta_{0}+\mu\right) u \eta+\mu u-\nu \eta_{x t}\right)_{x} & =0, \\
u_{t}+\beta_{0} \eta_{x}-\alpha_{0} u_{x x t}+\left(\left(\beta_{0}+\mu\right) u^{2} / 2+\mu \eta-\nu u_{x t}\right)_{x} & =0
\end{aligned}
$$

with $(\nu, \mu)$ close to 0 , and let us look for non trivial doubly periodic solutions in $H_{\text {ŁŁ }, 0}^{k, e} \times H_{\text {घh }}^{k, o}$. We observe that for $k \geq 2$

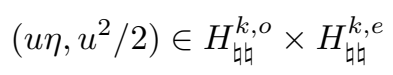

hence, defining $(f, g)$ by

$$
\begin{aligned}
& f=-\left(\beta_{0}+\mu\right) u \eta-\mu u+\nu \eta_{x t} \\
& g=-\left(\beta_{0}+\mu\right) u^{2} / 2-\mu \eta+\nu u_{x t}
\end{aligned}
$$

the right hand side of (5) has the properties required in lemma 3, once the compatibility condition is satisfied. We can then apply the Lyapunov-Schmidt method for finding the bifurcation equation. 


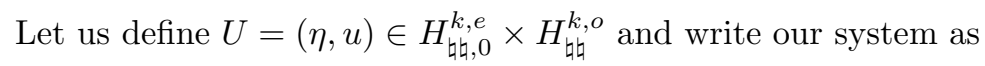

$$
\mathcal{L} U+\mu \mathcal{J} U-\nu U_{x t}+\left(\beta_{0}+\mu\right) \mathcal{N}(U, U)=0
$$

with

$$
\begin{aligned}
\mathcal{J} U & =\left(u,\left(1-\pi_{0}\right) \eta\right), \\
\mathcal{N}(U, U) & =\left(u \eta,\left(1-\pi_{0}\right) u^{2} / 2\right) .
\end{aligned}
$$

We observe that (14) is equivariant under the $O(2)$ symmetry defined above:

$$
\begin{aligned}
\mathcal{T}_{\tau} \mathcal{L} & =\mathcal{L T}_{\tau}, \mathcal{T}_{\tau} \mathcal{J}=\mathcal{J} \mathcal{T}_{\tau}, \mathcal{T}_{\tau} \mathcal{N}=\mathcal{N} \circ \mathcal{T}_{\tau} \\
\mathcal{S} \mathcal{L} & =-\mathcal{L} \mathcal{S}, \mathcal{S} \mathcal{J}=-\mathcal{J S}, \mathcal{S N}=-\mathcal{N} \circ \mathcal{S}
\end{aligned}
$$

Let us now decompose $U$ as follows (we are looking for real solutions)

$$
U=\Theta+\Upsilon
$$

with

$$
\begin{aligned}
\Theta & =A \xi_{0}+B \zeta_{0}+\overline{B \zeta_{0}} \\
\left(\Upsilon, \zeta_{0}\right) & =\left(\Upsilon, \overline{\zeta_{0}}\right)=\left(\Upsilon, \xi_{0}\right)=0
\end{aligned}
$$

where the scalar product is the one of $\left(L_{\natural t}^{2}\right)^{2}, A \in \mathbb{R}$ and $B \in \mathbb{C}$ are constants. We notice, as a consequence of the above decomposition, that $A$ is just the average of $\eta(x, t)$.

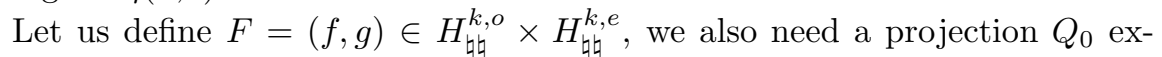
pressing the first two conditions in the compatibility condition (11) (since the third one is already satisfied by construction)

$$
Q_{0} F=F-\frac{1}{4 \pi^{2}}\left(F, \zeta_{1}\right) \zeta_{1}-\frac{1}{4 \pi^{2}}\left(F, \overline{\zeta_{1}}\right) \overline{\zeta_{1}}
$$

where we define

$$
\zeta_{1}=\left(e^{i q_{0} t} \sin p_{0} x, i e^{i q_{0} t} \cos p_{0} x\right)
$$

and we notice that the conditions $\left(F, \zeta_{1}\right)=\left(F, \overline{\zeta_{1}}\right)=0$ are required for $F$ to belong to the range of $\mathcal{L}$. We observe that

$$
\left(\zeta_{0}\right)_{x t}=-i p_{0} q_{0} \zeta_{1},\left(\zeta_{1}\right)_{x t}=i p_{0} q_{0} \zeta_{0}, \mathcal{J} \zeta_{0}=-i \zeta_{1}, \mathcal{J} \xi_{0}=0,
$$

hence

$$
Q_{0} \Theta_{x t}=0,\left(\Upsilon_{x t}, \zeta_{1}\right)=\left(\Upsilon_{x t}, \overline{\zeta_{1}}\right)=0, Q_{0} \mathcal{J} \Theta=0 .
$$

It results that (14) may be written as the system

$$
\begin{aligned}
& \mathcal{L} \Upsilon+\mu Q_{0} \mathcal{J}(\Upsilon)-\nu \Upsilon_{x t}+\left(\beta_{0}+\mu\right) Q_{0} \mathcal{N}(\Theta+\Upsilon, \Theta+\Upsilon)=0 \\
& \left(\mu \mathcal{J}(\Theta+\Upsilon)-\nu \Theta_{x t}+\left(\beta_{0}+\mu\right) \mathcal{N}(\Theta+\Upsilon, \Theta+\Upsilon), \zeta_{1}\right)=0 \\
& \left(\mu \mathcal{J}(\Theta+\Upsilon)-\nu \Theta_{x t}+\left(\beta_{0}+\mu\right) \mathcal{N}(\Theta+\Upsilon, \Theta+\Upsilon), \overline{\zeta_{1}}\right)=0 .
\end{aligned}
$$


Therefore, by considering (17) we get

$$
\Upsilon+\widetilde{\mathcal{L}}^{-1}\left\{\mu Q_{0} \mathcal{J}(\Upsilon)-\nu \Upsilon_{x t}+\left(\beta_{0}+\mu\right) Q_{0} \mathcal{N}(\Theta+\Upsilon, \Theta+\Upsilon)\right\}=0
$$

which is of the form

$$
\mathcal{F}(\Upsilon, A, B, \bar{B}, \mu, \nu)=0
$$

and thanks to the boundedness properties of the operator $\widetilde{\mathcal{L}}^{-1}$ shown at lemma $3, \mathcal{F}$ is analytic:

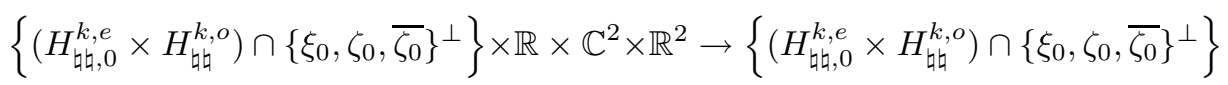

and satisfies

$$
\mathcal{F}(0, A, 0,0, \mu, \nu)=0
$$

and because of the fact that

$$
\begin{aligned}
& \mathcal{S} \zeta_{0}=\overline{\zeta_{0}}, \quad \mathcal{T}_{\tau} \zeta_{0}=e^{i q_{0} \tau} \zeta_{0} \\
& \mathcal{S} \xi_{0}=\xi_{0}, \quad \mathcal{T}_{\tau} \xi_{0}=\xi_{0},
\end{aligned}
$$

the equivariance properties $(15,16)$ of our system leads to

$$
\begin{aligned}
\mathcal{T}_{\tau} \mathcal{F}(\Upsilon, A, B, \bar{B}, \mu, \nu) & =\mathcal{F}\left(\mathcal{T}_{\tau} \Upsilon, A, e^{i q_{0} \tau} B, e^{-i q_{0} \tau} \bar{B}, \mu, \nu\right) \\
\mathcal{S} \mathcal{F}(\Upsilon, A, B, \bar{B}, \mu, \nu) & =-\mathcal{F}(\mathcal{S} \Upsilon, A, \bar{B}, B, \mu, \nu) .
\end{aligned}
$$

The above equation (20) is solvable with respect to $\Upsilon \in H_{\text {听, }}^{k, e} \times H_{\text {如 }}^{k, o}$ by the analytic implicit function theorem, for $A, B, \mu, \nu$ close enough to 0 in $\mathbb{R} \times \mathbb{C} \times \mathbb{R}^{2}$. We then obtain

$$
\Upsilon=\mathcal{Y}(A, B, \bar{B}, \mu, \nu)
$$

where $\Upsilon$ is analytic in its arguments and its principal part is given by

$$
\Upsilon=-\beta_{0} \widetilde{\mathcal{L}}^{-1} Q_{0} \mathcal{N}(\Theta, \Theta)+O\left\{(|\mu|+|\nu|)\|\Theta\|^{2}+\|\Theta\|^{3}\right\}
$$

and, because of the uniqueness of $\mathcal{Y}$ (comes from the implicit function theorem), we have for any real $\tau$

$$
\begin{aligned}
\mathcal{Y}(A, \bar{B}, B, \mu, \nu) & =\mathcal{S} \mathcal{Y}(A, B, \bar{B}, \mu, \nu) \\
\mathcal{Y}\left(A, e^{i q_{0} \tau} B, e^{-i q_{0} \tau} \bar{B}, \mu, \nu\right) & =\mathcal{T}_{\tau} \mathcal{Y}(A, B, \bar{B}, \mu, \nu) .
\end{aligned}
$$

In addition, we notice that, because of the existence of the family of trivial solutions $U=(A, 0)=A \xi_{0}$ of (14), we have for any $(A, \mu, \nu)$ close enough to 0

$$
\mathcal{Y}(A, 0,0, \mu, \nu)=0 .
$$

By simple calculation, we have

$$
\begin{aligned}
\mathcal{N}(\Theta, \Theta)= & \left(-i \sin p_{0} x\left(A B e^{i q_{0} t}-A \bar{B} e^{-i q_{0} t}\right)-\frac{i}{2}\left(B^{2} e^{2 i q_{0} t}-\bar{B}^{2} e^{-2 i q_{0} t}\right) \sin 2 p_{0} x,\right. \\
& \left.\frac{1}{4} \cos 2 p_{0} x\left(B^{2} e^{2 i q_{0} t}+\bar{B}^{2} e^{-2 i q_{0} t}\right)-\frac{1}{2}|B|^{2} \cos 2 p_{0} x\right)
\end{aligned}
$$


and

$$
\begin{aligned}
Q_{0} \mathcal{N}(\Theta, \Theta)= & \frac{1}{2}\left(-i \sin p_{0} x\left(A B e^{i q_{0} t}-A \bar{B} e^{-i q_{0} t}\right)-\frac{i}{2}\left(B^{2} e^{2 i q_{0} t}-\bar{B}^{2} e^{-2 i q_{0} t}\right) \sin 2 p_{0} x,\right. \\
& -\frac{1}{2} \cos p_{0} x\left(A B e^{i q_{0} t}+A \bar{B} e^{-i q_{0} t}\right)+\frac{1}{4} \cos 2 p_{0} x\left(B^{2} e^{2 i q_{0} t}+\bar{B}^{2} e^{-2 i q_{0} t}\right) \\
& \left.-\frac{1}{2}|B|^{2} \cos 2 p_{0} x\right) .
\end{aligned}
$$

The principal part of $\mathcal{Y}$ is then given by

$$
-\beta_{0} \widetilde{\mathcal{L}}^{-1} Q_{0} \mathcal{N}(\Theta, \Theta):=\left(y_{1}, y_{2}\right)
$$

with

$$
\begin{gathered}
y_{1}=\frac{1}{4} \cos p_{0} x\left(A B e^{i q_{0} t}+A \bar{B} e^{-i q_{0} t}\right)+\frac{1}{2}|B|^{2} \cos 2 p_{0} x+ \\
+\alpha_{1} \cos 2 p_{0} x\left(B^{2} e^{2 i q_{0} t}+\bar{B}^{2} e^{-2 i q_{0} t}\right) \\
y_{2}=\frac{i}{4} \sin p_{0} x\left(A B e^{i q_{0} t}-A \bar{B} e^{-i q_{0} t}\right)+i \beta_{1} \sin 2 p_{0} x\left(B^{2} e^{2 i q_{0} t}-\bar{B}^{2} e^{-2 i q_{0} t}\right)
\end{gathered}
$$

with

$$
\begin{aligned}
\alpha_{1} & =\frac{\beta_{0}\left(1+3 \alpha_{0} p_{0}^{2}\right)}{4 \alpha_{0} p_{0} q_{0}\left(2+5 \alpha_{0} p_{0}^{2}\right)}=\frac{\left(1+\alpha_{0} p_{0}^{2}\right)\left(1+3 \alpha_{0} p_{0}^{2}\right)}{4 \alpha_{0} p_{0}^{2}\left(2+5 \alpha_{0} p_{0}^{2}\right)} \\
\beta_{1} & =\frac{-\beta_{0}\left(1+2 \alpha_{0} p_{0}^{2}\right)}{4 \alpha_{0} p_{0} q_{0}\left(2+5 \alpha_{0} p_{0}^{2}\right)}=\frac{-\left(1+\alpha_{0} p_{0}^{2}\right)\left(1+2 \alpha_{0} p_{0}^{2}\right)}{4 \alpha_{0} p_{0}^{2}\left(2+5 \alpha_{0} p_{0}^{2}\right)} .
\end{aligned}
$$

Now, substituting $\Upsilon=\mathcal{Y}(A, B, \bar{B}, \mu, \nu)$ into (18) we obtain an equation in $\mathbb{C}$ of the form

$$
h(A, B, \bar{B}, \mu, \nu)=0
$$

while (19) gives its complex conjugate. Now, let us use the equivariance of our system. We then obtain the properties

$$
\begin{aligned}
h\left(A, B e^{i q_{0} \tau}, \bar{B} e^{-i q_{0} \tau}, \mu, \nu\right) & =e^{i q_{0} \tau} h(A, B, \bar{B}, \mu, \nu) \\
h(A, \bar{B}, B, \mu, \nu) & =-\overline{h(A, B, \bar{B}, \mu, \nu)}
\end{aligned}
$$

as it can be seen below. We have thanks to (15)

$$
\begin{aligned}
& h\left(A, B e^{i q_{0} \tau}, \bar{B} e^{-i q_{0} \tau}, \mu, \nu\right) \\
= & \left(\mu \mathcal{J} \mathcal{T}_{\tau}(\Theta+\mathcal{Y})-\nu \mathcal{T}_{\tau} \Theta_{x t}+\left(\beta_{0}+\mu\right) \mathcal{N}\left(\mathcal{I}_{\tau}(\Theta+\mathcal{Y}), \mathcal{T}_{\tau}(\Theta+\mathcal{Y})\right), \zeta_{1}\right) \\
= & \left(\mathcal{T}_{\tau}\left\{\mu \mathcal{J}(\Theta+\mathcal{Y})-\nu \Theta_{x t}+\left(\beta_{0}+\mu\right) \mathcal{N}(\Theta+\mathcal{Y}, \Theta+\mathcal{Y})\right\}, \zeta_{1}\right) \\
= & \left(\mu \mathcal{J}(\Theta+\Upsilon)-\nu \Theta_{x t}+\left(\beta_{0}+\mu\right) \mathcal{N}(\Theta+\Upsilon, \Theta+\Upsilon), \mathcal{T}_{-\tau} \zeta_{1}\right) \\
= & e^{i q_{0} \tau}\left(\mu \mathcal{J}(\Theta+\Upsilon)-\nu \Theta_{x t}+\left(\beta_{0}+\mu\right) \mathcal{N}(\Theta+\Upsilon, \Theta+\Upsilon), \zeta_{1}\right) \\
= & e^{i q_{0} \tau} h(A, B, \bar{B}, \mu, \nu)
\end{aligned}
$$


and thanks to (16)

$$
\begin{aligned}
& h(A, \bar{B}, B, \mu, \nu) \\
= & \left(\mu \mathcal{J} S(\Theta+\mathcal{Y})-\nu \mathcal{S} \Theta_{x t}+\left(\beta_{0}+\mu\right) \mathcal{N}(\mathcal{S}(\Theta+\mathcal{Y}), \mathcal{S}(\Theta+\mathcal{Y})), \zeta_{1}\right) \\
= & -\left(\mathcal{S}\left\{\mu \mathcal{J}(\Theta+\mathcal{Y})-\nu \Theta_{x t}+\left(\beta_{0}+\mu\right) \mathcal{N}(\Theta+\mathcal{Y}, \Theta+\mathcal{Y})\right\}, \zeta_{1}\right) \\
= & -\left(\mu \mathcal{J}(\Theta+\mathcal{Y})-\nu \Theta_{x t}+\left(\beta_{0}+\mu\right) \mathcal{N}(\Theta+\mathcal{Y}, \Theta+\mathcal{Y}), \overline{\zeta_{1}}\right) \\
= & -\overline{h(A, B, \bar{B}, \mu, \nu) .}
\end{aligned}
$$

It results from its analyticity, that $h$ takes the form

$$
h(A, B, \bar{B}, \mu, \nu)=i B H\left(A,|B|^{2}, \mu, \nu\right)
$$

with a analytic function $H$ taking only real values, and the complex equation $h=0$ reduces to either $B=0$ or the real equation $H=0$. Now noticing that

$$
\begin{aligned}
\left(\Theta_{x t}, \zeta_{1}\right) & =-i 4 \pi^{2} p_{0} q_{0} B \\
\left(\mathcal{N}(\Theta, \Theta), \zeta_{1}\right) & =-i 2 \pi^{2} A B \\
\left(\mathcal{J} \Theta, \zeta_{1}\right) & =-i 4 \pi^{2} B
\end{aligned}
$$

the bifurcation equation (18) reads

$$
i B H\left(A,|B|^{2}, \mu, \nu\right)=0
$$

where

$-\left(4 \pi^{2}\right)^{-1} H\left(A,|B|^{2}, \mu, \nu\right)=\mu-p_{0} q_{0} \nu+\frac{1}{2} \beta_{0} A-\beta_{2}|B|^{2}+O\left(|B|^{4}+(|\mu|+|\nu|+|A|)\left(|A|+|B|^{2}\right)\right)$

and the term which is the most important to compute is the coefficient $4 i \pi^{2} \beta_{2}$ in (22). For this we need to introduce the symmetric bilinear operator $\mathcal{N}$ by

$$
2 \mathcal{N}\left(U_{1}, U_{2}\right)=\left(u_{1} \eta_{2}+u_{2} \eta_{1},\left(1-\pi_{0}\right) u_{1} u_{2}\right)
$$

Since we have

$$
y:=\left(y_{1}, y_{2}\right):=A B y_{p_{0}, q_{0}}+A \bar{B} y_{p_{0},-q_{0}}+|B|^{2} y_{2 p_{0}, 0}+B^{2} y_{2 p_{0}, 2 q_{0}}+\bar{B}^{2} y_{2 p_{0},-2 q_{0}}
$$

we then obtain

$$
\beta_{2}=\frac{\beta_{0}}{4 i \pi^{2}}\left(2 \mathcal{N}\left(y_{2 p_{0}, 0}, \zeta_{0}\right)+2 \mathcal{N}\left(y_{2 p_{0}, 2 q_{0}}, \overline{\zeta_{0}}\right), \zeta_{1}\right)
$$

therefore,

$$
\beta_{2}=\beta_{0}\left(\frac{1}{8}+\frac{\beta_{1}}{2}-\frac{\alpha_{1}}{4}\right)=\frac{3 \beta_{0}\left[\left(\alpha_{0} p_{0}^{2}-1\right)^{2}-2\right]}{16 \alpha_{0} p_{0}^{2}\left(2+5 \alpha_{0} p_{0}^{2}\right)} .
$$

We can in addition give the exact term $H(A, 0, \mu, \nu)$ independent of $B$. For this, let us look for all terms of degree one in $B$, and degree 0 in $\bar{B}$ in the expression $h(A, B, \bar{B}, \mu, \nu)$. Due to the form of $\mathcal{Y}(A, B, \bar{B}, \mu, \nu)$, these terms come from

$$
\left(\mu \mathcal{J}\left(B \zeta_{0}+Y_{B}\right)-\nu B \zeta_{0, x t}+\left(\beta_{0}+\mu\right)\left\{2 \mathcal{N}\left(A \xi_{0}, B \zeta_{0}\right)+2 \mathcal{N}\left(A \xi_{0}, Y_{B}\right)\right\}, \zeta_{1}\right)
$$


where $Y_{B}$ is the term in $\mathcal{Y}(A, B, \bar{B}, \mu, \nu)$ of degree one in $B$, and degree 0 in $\bar{B}$. Now, $Y_{B}$ is the solution of the affine equation

$$
\mathcal{L} Y_{B}+\mu Q_{0} \mathcal{J}\left(Y_{B}\right)-\nu Y_{B, x t}+\left(\beta_{0}+\mu\right) Q_{0}\left\{2 \mathcal{N}\left(A \xi_{0}, B \zeta_{0}\right)+2 \mathcal{N}\left(A \xi_{0}, Y_{B}\right)\right\}=0
$$

and a careful examination shows that we can look for $Y_{B}$ under the form

$$
Y_{B}=B \gamma(A)\left(e^{i q_{0} t} \cos p_{0} x, i e^{i q_{0} t} \sin p_{0} x\right) .
$$

A direct identification in (25) leads to

$$
\left(2 \beta_{0}+\mu+p_{0} q_{0} \nu\right) \gamma(A)-\frac{1}{2}\left(\beta_{0}+\mu\right) A\{1-\gamma(A)\}=0,
$$

which gives $\gamma(A)$ :

$$
\begin{aligned}
\gamma_{\mu, \nu}(A) & =\frac{\left(\beta_{0}+\mu\right) A}{2\left(2 \beta_{0}+\mu+p_{0} q_{0} \nu\right)+\left(\beta_{0}+\mu\right) A} \\
& =\frac{\beta A}{2\left(2 \beta-\mu+p_{0} q_{0} \nu\right)+\beta A},
\end{aligned}
$$

which is coherent (taking the limit $\mu, \nu, A$ tending towards 0 ) with the coefficient $y_{p_{0}, q_{0}}$ of $A B$ in $\left(y_{1}, y_{2}\right)$. We then observe that

$$
\left(\mathcal{J}\left(Y_{B}\right), \zeta_{1}\right)=0
$$

which leads for the coefficient (24) to the following expression

$$
-4 i \pi^{2} B\left\{\mu-p_{0} q_{0} \nu+\frac{1}{2}\left(\beta_{0}+\mu\right) A\left(1-\gamma_{\mu, \nu}(A)\right)\right\} .
$$

Now, from the form of $\gamma_{\mu, \nu}(A)$, and from the identity

$$
\mu-p_{0} q_{0} \nu=\frac{1}{p_{0}}\left(p_{0} \beta-q_{0}\left(1+\alpha p_{0}^{2}\right)\right.
$$

we obtain

$$
\mu-p_{0} q_{0} \nu+\frac{1}{2}\left(\beta_{0}+\mu\right) A\left(1-\gamma_{\mu, \nu}(A)\right)=\frac{2\left\{p_{0}^{2} \beta^{2}(1+A)-q_{0}^{2}\left(1+\alpha p_{0}^{2}\right)^{2}\right\}}{p_{0}\left\{2\left(p_{0} \beta+q_{0}\left(1+\alpha p_{0}^{2}\right)\right)+p_{0} \beta A\right\}} .
$$

We then obtain, in addition to the trivial family of solutions of (22) corresponding to $B=0$ (already seen), another bifurcating family given by the solutions of $H\left(A,|B|^{2}, \mu, \nu\right)=0$ i.e. thanks to the analyticity of $H$ and the above computation, the solutions of the following improved form for (23)

$$
\frac{2\left\{p_{0}^{2} \beta^{2}(1+A)-q_{0}^{2}\left(1+\alpha p_{0}^{2}\right)^{2}\right\}}{p_{0}\left\{2\left(p_{0} \beta+q_{0}\left(1+\alpha p_{0}^{2}\right)\right)+p_{0} \beta A\right\}}-\beta_{2}|B|^{2}+O\left(|B|^{4}+(|\mu|+|\nu|+|A|)\left(|B|^{2}\right)\right)=0 .
$$


This provides standing waves, determined up to a phase shift in $t$, equivalent to an arbitrary choice of the phase of $B$. Moreover, for $\beta_{2} \neq 0$, we can solve, via the implicit function theorem, with respect to $|B|^{2}$, and

$$
|B|^{2}=\frac{p_{0}^{2} \beta^{2}(1+A)-q_{0}^{2}\left(1+\alpha p_{0}^{2}\right)^{2}}{2 p_{0}^{2} \beta_{0} \beta_{2}}\{1+O(|A|+|\mu|+|\nu|)\}
$$

for arbitrary $A, \mu, \nu$ close to 0 , while the bifurcation only takes place either for $p_{0}^{2} \beta^{2}(1+A)-q_{0}^{2}\left(1+\alpha p_{0}^{2}\right)^{2}>0$ or for $p_{0}^{2} \beta^{2}(1+A)-q_{0}^{2}\left(1+\alpha p_{0}^{2}\right)^{2}<0$. We sum up our result in the following

Theorem 4 Consider any positive $\left(\alpha_{0}, \beta_{0}\right)$ such that

$$
\Sigma_{\left(\alpha_{0}, \beta_{0}\right)}=\left\{(p, q) \mid(p, q) \in \mathbb{N}^{2} \text { and } q\left(1+\alpha_{0} p^{2}\right)-p \beta_{0}=0\right\}
$$

has a unique element $\left(p_{0}, q_{0}\right)$. Then, for $\mu, \nu, A$ close enough to 0 , where $\alpha=$ $\alpha_{0}+\nu, \beta=\beta_{0}+\mu, A$ is the average of $\eta(x, t)$, and for

$$
\left\{\left(p_{0}^{2} \beta^{2}(1+A)-q_{0}^{2}\left(1+\alpha p_{0}^{2}\right)^{2}\right\}\left(\alpha_{0} p_{0}^{2}-(1+\sqrt{2})\right)>0,\right.
$$

there is a three parameter $(\alpha, \beta, A)$ family of bifurcating standing waves $U=$ $(\eta, u)$, solution of the system (3,4) in $H_{\text {听, }}^{k, e} \times H_{\text {响 }}^{k, o}: \mathcal{T}_{\tau} U_{0}, \tau \in \mathbb{R}$, and

$$
\begin{aligned}
U_{0}(x, t) & =(A, 0)+2|B|\left(\cos q_{0} t \cos p_{0} x, \sin q_{0} t \cos p_{0} x\right)+O(|B|(|A|+|B|)) \\
|B|^{2} & =\frac{p_{0}^{2} \beta^{2}(1+A)-q_{0}^{2}\left(1+\alpha p_{0}^{2}\right)^{2}}{2 p_{0}^{2} \beta_{0} \beta_{2}}\{1+O(|A|+|\mu|+|\nu|)\} .
\end{aligned}
$$

Remark: If we consider the linearization of the system (3)(4) at a point $(\eta, u)=(A, 0)$ instead of the origin, we obtain for the inverse operator, a new denominator replacing $\Delta$ in (6) which is

$$
q^{2}\left(1+\alpha p^{2}\right)^{2}-p^{2} \beta^{2}(1+A) .
$$

This is the quantity appearing in the expression of the amplitude of the bifurcating standing waves, as it is usual. Now, the set of $(\alpha, \beta, A)$ in the threeparameter space, where bifurcation takes place is when the above expression cancels, which is for fixed $(p, q)$ a right conoid (axis: $\alpha=-1 / p^{2}, \beta=0$ ) called a Whitney's umbrella. The intersection of this surface with the plane $A=0$ is the couple of straight lines already mentioned in the remark of section 2 . The above theorem shows that the bifurcation of standing waves takes place along a discrete set of such Whitney's umbrellas (don't forget that $(p, q)$ is arbitrary in $\mathbb{N}^{2}$ ).

\section{References}

[1] J. P. Albert, A. Alazman, J. L. Bona, M. Chen, And J. Wu, Comparisons between the BBM equation and a Boussinesq system, preprint (2002). 
[2] J. L. Bona And M. Chen, A Boussinesq system for two-way propagation of nonlinear dispersive waves, Physica D, 116 (1998), pp. 191-224.

[3] J. L. Bona, M. Chen, And J.-C. SAUt, Boussinesq equations and other systems for small-amplitude long waves in nonlinear dispersive media I: Derivation and the linear theory, J. Nonlinear Sci., 12 (2002), pp. 283-318.

[4] — Boussinesq equations and other systems for small-amplitude long waves in nonlinear dispersive media II: Nonlinear theory, Nonlinearity, 17 (2004), pp. $925-952$.

[5] J. L. Bona, T. Colin, And D. LAnnes, Long wave approximations for water waves, preprint (2004).

[6] J. L. Bona, W. G. Pritchard, And L. R. Scott, An evaluation of a model equation for water waves, Philos. Trans. Royal Soc. London, Ser. A, 302 (1981), pp. 457-510.

[7] W. Craig, An existence theory for water waves, and Boussinesq and Korteweg-de Vries scaling limits, Comm. PDE, 10 (1985), pp. 787-1003.

[8] G.Iooss, P.Plotnikov, J.Toland. Standing waves on an infinitely deep perfect fluid under gravity. Preprint 2004.

[9] P.I.Plotnikov, J.F.Toland. Nash-Moser theory for standing waves. Arch. Rat. Mech. Anal. 159 (2001), 1-83.

[10] G. Schneider And C. G. Wayne, The long-wave limit for the water wave problem. I. The case of zero surface tension, Comm. Pure Appl. Math., 53 (2000), pp. 1475-1535. 